\title{
Improving soil water representation in the Australian Water Resources Assessment landscape model through the assimilation of remotely-sensed soil moisture products
}

\author{
$\underline{\text { L.J. Renzullo }}^{\text {a }}$, D. Collins ${ }^{\text {b }}$, J.-M. Perraud ${ }^{\text {a }}$, B. Henderson ${ }^{\text {c }}$, H. Jin ${ }^{\text {c }}$ and A. Smith ${ }^{\text {d }}$ \\ ${ }^{a}$ CSIRO Land and Water, Canberra, Australian Capital Territory 2601 \\ ${ }^{b}$ CSIRO Advanced Scientific Computing, Kensington, Western Australia \\ ${ }^{c}$ CSIRO Computational Informatics, Canberra, Australian Capital Territory, 2601 \\ ${ }^{d}$ Bureau of Meteorology, Melbourne, Victoria \\ Email: Luigi.Renzullo@csiro.au
}

\begin{abstract}
Many researchers over the last decade have demonstrated how the assimilation of satellite soil moisture data can improve the accuracy of soil water representation in land surface models, and result in improved estimates of evaporative flux, drainage, and runoff. In this study we investigated whether similar benefits are achievable for the Australian Water Resources Assessment landscape (AWRA-L) model through the assimilation of AMSR-E and ASCAT soil moisture (SM) products.
\end{abstract}

The AWRA-L model was co-developed by CSIRO and Bureau of Meteorology researchers to support the Bureau's mandated reporting requirements on national water accounts and water resource assessments. AWRA-L represents the soil column as three conceptual storage layers: a top-layer (equivalent to the emitting soil layer for C- or L-band microwave radiometry); and separate layers for shallow- and deep-rooted vegetation respectively. AWRA-L was run cell-wise (i.e. no lateral flow) across the continent at 0.05-degree resolution providing estimates of daily water fluxes and stores.

We used perturbed meteorological forcing and the ensemble Kalman filter (EnKF) to assimilate AMSR-E and ASCAT SM products into AWRA-L. Evaluations to-date have been conducted using the OzNet network of in situ moisture sensors, but will be extended to other parts of the continent via the network of cosmic ray probes and (indirectly) through evaluation of independent satellite SM retrievals. Preliminary results clearly show an improvement in AWRA-L top-layer SM estimation compared to open-loop simulations. Results for the impact on root-zone soil layers are mixed, but appear to be linked to combinations of prescribed SM error and/or strength of vertical coupling between soil layers. Further investigations will identify where and when the assimilation of satellite SM benefits AWRA-L estimation in terms of soil water status and runoff estimation across Australia.

Keywords: Data assimilation, satellite soil moisture, shallow root-zone 


\section{INTRODUCTION}

Many researchers over the past decade have demonstrated that the assimilation of remotely-sensed soil moisture products into land surface models can improve the soil water representation of these models and result in improved estimates of evaporative fluxes, drainage and runoff (e.g. Reichle and Koster, 2005; Brocca et al., 2010; Draper et al., 2012). The ability to constrain water balance estimation over large areas offers great potential for continental water resources assessment and management, particularly in parts of the landscape where traditional networks of data constraint (e.g. streamflow data) have sparse/intermittent coverage.

In this study we investigate whether the assimilation of satellite soil moisture products into a landscape water balance model leads to improved root-zone soil moisture estimation. The model used (described in Section 2.1) is part of a larger modelling system designed to augment surface metering where available, and provide comprehensive coverage of key water balance terms for national-scale water resources assessment. Evaluating root-zone soil moisture estimation may be considered one of the first steps towards quality assurance of the model performance, as soil moisture is a key variable in the partitioning of rainfall into evaporation, infiltration and runoff.

While both the satellite soil moisture data (described in Section 3.1) and the water balance modeling are continental in extent, our evaluations are limited to southeastern Australia and a ground-based network of soil moisture sensors (described in Section 3.3). We present the results of the evaluation of the assimilation results against the in situ data, and improvement is assessed against the unconstrained model estimates.

\section{METHOD}

\subsection{The Australian Water Resources Assessment landscape model: AWRA-L}

The Australian Water Resources Assessment (AWRA) system was developed under the Water Information Research and Development Alliance (WIRADA). For the investigation we limited our focus to the landscape model component of the AWRA system: AWRA-L (Van Dijk 2010). AWRA-L described the time evolution of water stores and fluxes across a region of interest. Gridded forcing data (spatial estimates of meteorological observations, see Section 2.2) drive the model to spatial water balance estimates on a grid of $0.05^{\circ} \times 0.05^{\circ}$ cells across the region. Note that each cell is modelled independently of its neighbours (i.e. there is no lateral transport of water).

The AWRA-L state variables, denoted in the following section as $X$, are the soil water storages in the top soil

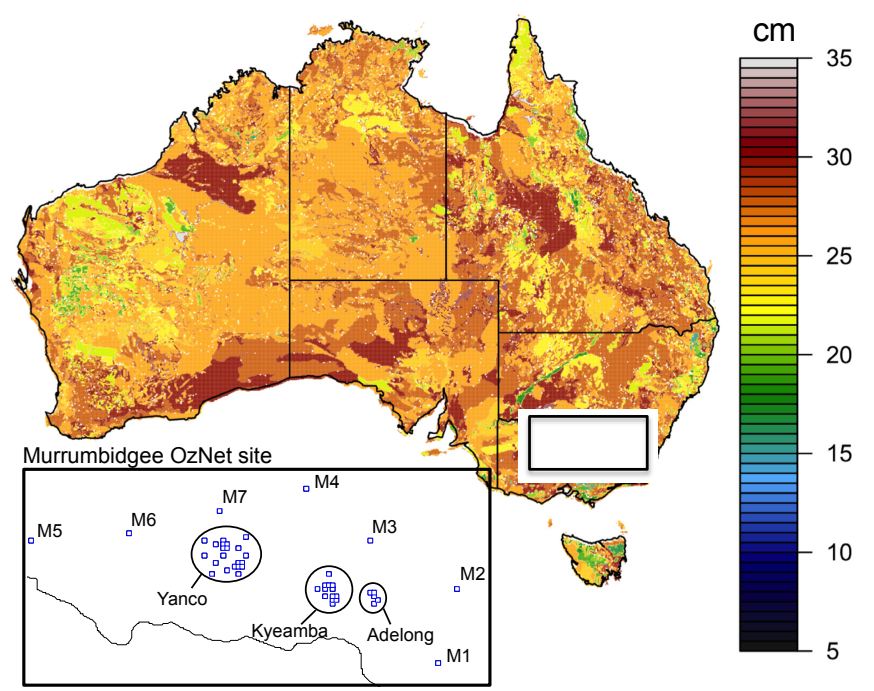

Figure 1. Geographic variation in AWRA-L shallow rootzone soil depth. Inset shows the OzNet soil moisture monitoring sites used in the evaluation of this work. layer (closest conceptually to the emitting soil layer for C- or L-band microwave radiometry, although may be up to 4 times thicker), a shallow-rooted vegetation layer and a deep-rooted vegetation layer. For the AWRA-L model used in this study, we use the ASRIS soil information (http://www.asris.csiro.au) to infer soil layer thicknesses for the top, shallow-root and deep-root layers to be between 7-9 cm, 18-21 cm and $6-9 \mathrm{~m}$ respectively. This means that a shallow root-zone for the AWRA-L model can be defined as broadly described as between $0-30 \mathrm{~cm}$, but of course does vary geographically (Figure 1).

The remotely-sensed soil moisture data used in this study are represented as $Y$. These data, and the surface network of moisture monitoring probes used in the following evaluations (Figure 1 inset), are described further in Section 3 


\subsection{Data assimilation method}

In this study we chose the ensemble Kalman filter (EnKF, Evensen, 2003) approach to assimilate satellite soil moisture into AWRA-L The EnKF is a popular technique for assimilating satellite soil moisture into land surface models, and is suitable for use with moderately nonlinear models. It uses ensembles of model states to derive error statistics needed to optimally combine models with noisy observations. In the following we provide only a summary of the salient features of the assimilation approach; details of the method and the application are provided elsewhere (Renzullo et al., in prep.; Perraud et al., in these proceedings).

Common to all filter-based assimilation techniques are: a forecast step, where model states from the previous time step are propagated forward to the current time step by forcing the dynamic model with meteorological data; and an analysis or update step, where forecast states are adjusted towards available observation by an increment dependent on the size of model and observation error variances.

\section{Forecast step: perturbed meteorological forcing}

We used perturbed meteorology forcing data to drive AWRA-L and generate ensembles of forecast model states at time $t$, denote $\left\{X_{t}^{i, f}\right\}_{i=1}^{N e}$, where $N e$ is the number of ensemble members. The meteorological data used were those from the Australian Water Availability Project (AWAP) as detailed in Jones et al. (2009). Our choice of errors for the meteorological data was guided by the reported evaluation statistics (Jones et al., 2009) and our own anecdotal findings. For daily incoming shortwave radiation we used an additive error of $50 \mathrm{Wm}^{-2}$ and for air temperature (derived from AWAP product daily TMIN and TMAX) we used an error of $2.5 \mathrm{~K}$. For rainfall, which has the greatest impact on ensemble spread, we used a relative error of $40 \%$. We acknowledge that for most large parts of Australia this is an underrepresentation of the error (particularly in gauge-sparse regions). However we consider it appropriate for our study area. Note that for rainfall we have used a multiplicative error, as opposed to the additive error for radiation and air temperature. This avoids the problem of perturbations resulting in negative rainfall values when low or zero rainfall is recorded.

\section{Analysis step: state updating}

Given satellite soil moisture observations, $Y_{t}$, with specified error variance $R$, ensembles of forecast AWRAL states, $\left\{X_{t}^{i, f}\right\}_{i=1}^{N e}$ and observation operator, $h$, we compute the analysis states as,

$$
X_{t}^{i, a}=X_{t}^{i, f}+P_{t}^{f} H^{T}\left(H P_{t}^{f} H^{T}+R\right)^{-1}\left[Y_{t}-h\left(X_{t}^{i, f}\right)+\varepsilon_{i}\right]
$$

where $\varepsilon_{i} \sim N(0, R)$. The ensembles are used to estimate error variances/covariance matrices $P_{t}^{f} H^{T} \approx$ $\operatorname{cov}\left(\left\{X_{t}^{i, f}\right\}_{i=1}^{N e},\left\{h\left(X_{t}^{i, f}\right)\right\}_{i=1}^{N e}\right)$ and $H P_{t}^{f} H^{T} \approx \operatorname{cov}\left(\left\{h\left(X_{t}^{i, f}\right)\right\}_{i=1}^{N e}\right)$, respectively.

The degree of influence of the observation on the model states is related to the relative magnitudes of observation and model error variance matrices: e.g. as $R$ tends towards zero, the analysis states become dominated by the observation. Alternatively if $H P_{t}^{f} H^{T}<<\mathrm{R}$, observations have very little or no influence on model estimation. A common problem with ensemble-based assimilation is the collapse of the ensemble spread, i.e. $H P_{t}^{f} H^{T}=0$, due to the reuse of a small number of ensemble members between time steps. Here we employed both 'double EnKF' and covariance inflation techniques (see Evensen 2003 for definitions) to minimize the occurrence of ensemble collapse. We note that for EnKF to work optimally, care must be taken to ensure that the ensembles adequately represent the model error by having sufficient spread so that the observations exert constraint, but not too large to result in overfitting.

\section{DATA SETS AND OBSERVATION PRE-PROCESSING}

\subsection{Satellite soil moisture}

The first soil moisture (SM) data set used in our investigations is based on the Advanced Microwave Scanning Radiometer for the Earth Observing System (AMSR-E) aboard the Aqua satellite and derived via the retrieval algorithm of Owe et al. (2008). To facilitate the assimilation into the AWRA-L model these data were resampled (using nearest-neighbour) from their original 0.25-degree resolution to the AWRA-L modelling grid of 0.05-degree resolution for Australia. The daily SM estimates used here are derived from AMSR-E descending passes, which correspond to "nighttime" overpass locally. Our data holdings for this product range from 1 July 2002 - 30 September 2011. 
The second SM data used are derived from Advanced Scatterometer (ASCAT) data aboard the MetOp-A satellite using the change detection algorithm of Wagner et al. (1999). Specifically the data are the Surface Degree of Saturations (SDS) values ranging from 0 (driest conditions on record) to $100 \%$ (wettest conditions on record). Like the AMSR-E product, the data are derived from C-band measurements and therefore correspond to top $\sim 2 \mathrm{~cm}$ of surface soil. As with the AMSR-E SM product the ASCAT SDS data were remapped to the AWRA 0.05-degree resolution from the original 0.125-degree data using nearest-neighbour resampling. Both ascending and descending passes of SDS data are averaged following the approach of Liu et al. (2011) to generate the "daily" ASCAT SM data used in the investigation. Our ASCAT data holdings range from 1 Jan 2007 - 31 Dec 2011.

\subsection{Observation 'bias correction' and error characterisation}

A standard data pre-processing step of data assimilation algorithms is the rescaling of the SM observations to remove any systematic difference between model and observed time series (the so-called bias correction). Rescaling SM values minimises potentially detrimental influences of the observations on the model due to inconstancy in the definition of SM (Koster et al., 2009) by transforming the observations into model space. We use linear cumulative distribution function (CDF) matching to rescale the satellite SM values pixel-wise for the whole country. CDF matching ensures that the statistical distribution of both the satellite and AWRA$\mathrm{L}$ model time series are the same, and that assimilation adjusts the model for random variations.

The triple collocation (TC) has become somewhat of an 'industry standard' for generating spatially explicit error estimates for SM data. The technique allows the simultaneous pixel-wise estimation of the error structure, and the cross-calibration of a set of at least three datasets under the assumption that the datasets are linearly related and that the errors are uncorrelated. Here we have used the approach of Caires \& Sterl (2003). The technique is applied to the bias corrected SM data to provide AMSR-E and ASCAT error estimates respectively, with AWRA-L SM used as the reference product. TC errors for the SM products range from 5 $15 \%(10 \%$ on average $)$, with general increasing west to east trend.

\subsection{Surface soil moisture measurements}

Surface observations used to verify our modelling results were obtained from the OzNet Murrumbidgee network of in situ SM probes (Smith et al., 2012). The OzNet soil moisture monitoring network is comprised largely of TDR probes measuring volumetric SM at various soil depths at 63 locations across the Murrumbidgee catchment area (Figure 1). Specifically, we used 0-5 cm measurements to evaluate the model top-layer soil moisture, and the $0-30 \mathrm{~cm}$ measurements to evaluate the modeled shallow root-zone soil moisture. Note that only root-zone moisture measurements are only available for 38 of the OzNet sites.

For each OzNet probe, we computed daily soil moisture by taking the average of all the measurements (at 20 or 30 minute intervals) within the 24-hour period to 9am local time to be consistent with the AWAP rainfall forcing data. We assume these daily measurements to be representative of the moisture field for the coincident AWRA-L model cells; where a cell contained two or more probes, we used the arithmetic mean of the in situ measurements for that cell. In total, there are in situ data for 45 sites to evaluate top-layer soil moisture estimates and 37 sites for the shallow root-zone estimates. Finally, at the time of writing this paper, we only had access to the publicly available OzNet data, which end May 2011.

\section{RESULTS}

Here we evaluate the results of assimilating the bias-corrected AMSR-E and ASCAT SM data into AWRA-L against the OzNet in situ SM data. The CDF matched data were used along with the corresponding TC error estimate for both SM products, respectively. No quality control was applied to the satellite SM products.

AWRA-L simulations were conducted cell-wise (independent cells) across Australia at 0.05-degree intervals. This required extensive use of CSIRO's parallel computing infrastructure and large volumes $>1 \mathrm{~Tb}$ of disk storage. Details of this implementation are given in Perraud et al. (these proceedings). While this may seem excessive for the purposes of this investigation, the experiments were part of a large continental scale evaluation of AWRA-L's performance for other parts of the water balance.

AWRA-L simulations ran unconstrained, driven only with perturbed forcing, from 1 Jan 2000 to 31 Dec 2005. Six years of model spin-up was chosen after preliminary experimentation revealed that it was of sufficient length for some of the water balance terms (notably ground water and river water stores) and the ensemble-derived error statistics to be free from the influence of initial conditions. Assimilation commences 1 Jan 2006 and ends on 31 Dec 2011. 


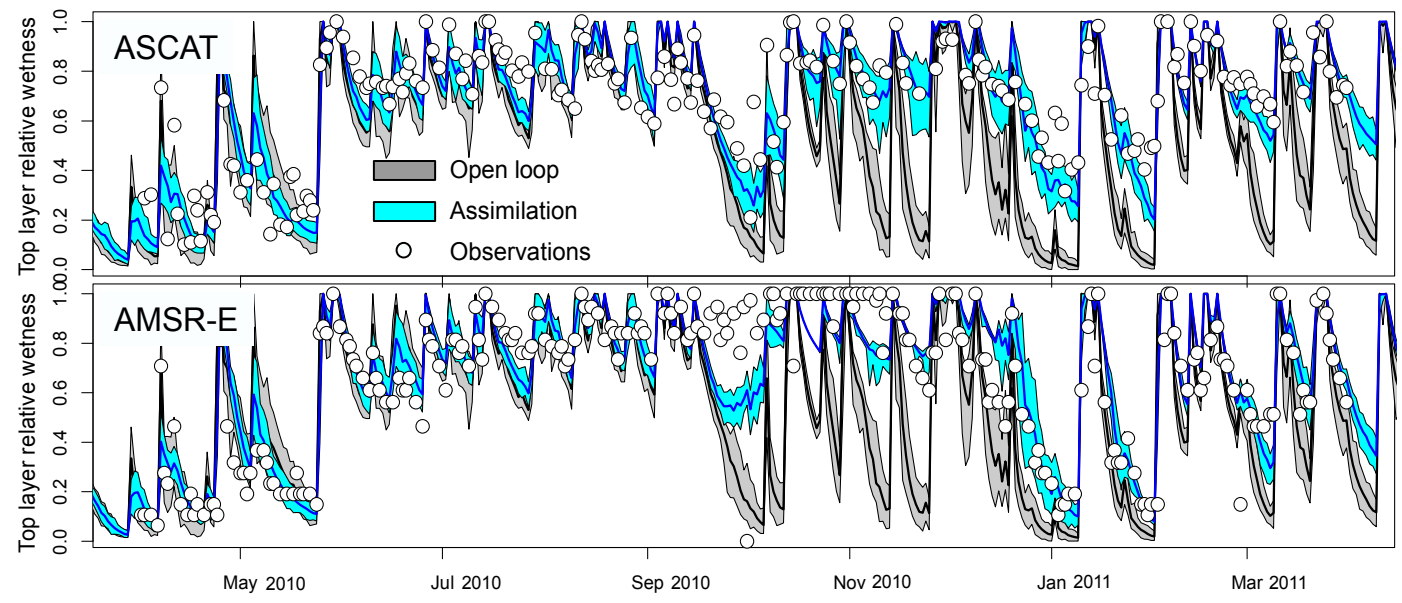

Figure 2. Time series of AWRA-L simulations compared to satellite SM estimates (white dots) for a Yanco OzNet site Y3. Ensemble median and range for open-loop simulations highlighted as a black line and grey shaded area, respectively. Analysis estimates after the assimilation of ASCAT and AMSR-E data in top and bottom panels respectively, with ensemble median and range as solid blue line and cyan shading.

Four experiments were conducted: assimilation of AMSR-E alone; assimilation of ASCAT alone; joint assimilation of AMSR-E and ASCAT; and open-loop (no assimilation) to serve as a reference. Given ASCAT SM time series begins in 1 Jan 2007, we evaluated the results of all experiments over the common period 1 July 2007 - 30 Sept 2011.

For each time step, 100 member ensembles of AWRA-L soil water storages were generated. Ensembles are summarised for output by their $0^{\text {th }}, 25^{\text {th }}, 50^{\text {th }}$ (median), $75^{\text {th }} 100^{\text {th }}$ percentiles and ensemble mean. Only the top-layer and shallow root-zone soil water storages are considered for the present investigation. To illustrate, Figure 2 depicts the ensemble median of AWRA-L model estimates (explained in the next paragraph) as solid lines (open-loop and analysis estimates in black and blue, respectively) and the ensemble range $\left(0^{\text {th }}\right.$ $100^{\text {th }}$ percentiles) as shaded regions (open-loop and analysis in grey and cyan, respectively).

Figure 2 provides examples of AWRA-L top-layer relative wetness estimates (i.e. top-layer waters storage, $S 0$, normalized by a field capacity storage parameter prescribed $a$ priori) with and without assimilation. The model output is displayed for 1 Apr 2010 - 30 Apr 2011 for a Yanco site in the Murrumbidgee (Figure 1). For comparison, the CDF matched ASCAT and AMSR-E SM data for the sites are displayed as white dots in the top and bottom panels respectively. The AWRA-L open-loop simulations can be seen to match the peaks in SM detected by both satellite data sets. However the model tends to dry out the top soil layer quicker than the observations suggest, particularly noticeable Oct 2010 - Jan 2011 period. After assimilating ASCAT and AMSR-E data into AWRA-L the analysis estimates were in closer agreement with the satellite values.

Quantitative evaluation of the AWRA-L assimilation results were based on spatially and temporally aggregated OzNet data (described in Section 3.3). The number of data for the comparison varies between sites: the older network has between 600 - 1380 data points over our evaluation period; the newer probes around the Yanco township in New South Wales have around 400-500 (a couple of sites having fewer than 100 points). Note that due to the incongruence of what AWRA-L models (water storage or relative wetness) and what the OzNet probes measure (volumetric SM), we limit our comparison to calculating the correlation of point time series, acknowledging that converting AWRA output into volumetric units, say, requires a linear transformation and therefore will not affect correlation but may introduce bias through the dependence on auxiliary soil hydraulic property information. Furthermore, we make our assessment of improvement in SM estimation with reference to open-loop simulations.

Ensemble mean top-layer SM estimates are evaluated against in situ 0-5 cm data at $45 \mathrm{OzNet}$ sites (Figure 3). Figure 3 (top panel) shows plots of correlation for the open-loop $\left(r^{0}\right)$ and the analysis $\left(r^{a}\right)$ estimates after the assimilation of (a) AMSR-E, (b) ASCAT and (c) both data sets jointly. Points falling above the one-to-one line indicate improved correlation relative to open-loop. Generally we see that assimilation has improved model estimation, with correlation increasing for $69 \%$ of sites with AMSR-E and $89 \%$ of sites with ASCAT. 
When there is degraded correlation as a result of assimilation it is typically no more than $6 \%$. The joint assimilation of satellite products also improves AWRA-L estimation (for $87 \%$ of site), which includes many of the sites where AMSR-E alone had resulted in slightly degraded correlation. Most notable improvements are seen at the lower correlation end where higher than 20-30\% improvement in correlation is achieved through SM assimilation. The sites of the greatest improvements correspond to the newer OzNet sites (around Yanco), however improvements are also seen at the M6, Y6 and A5 sites. At these sites the satellite SM data have low errors $(0.05-0.08$ in relative wetness units). We also
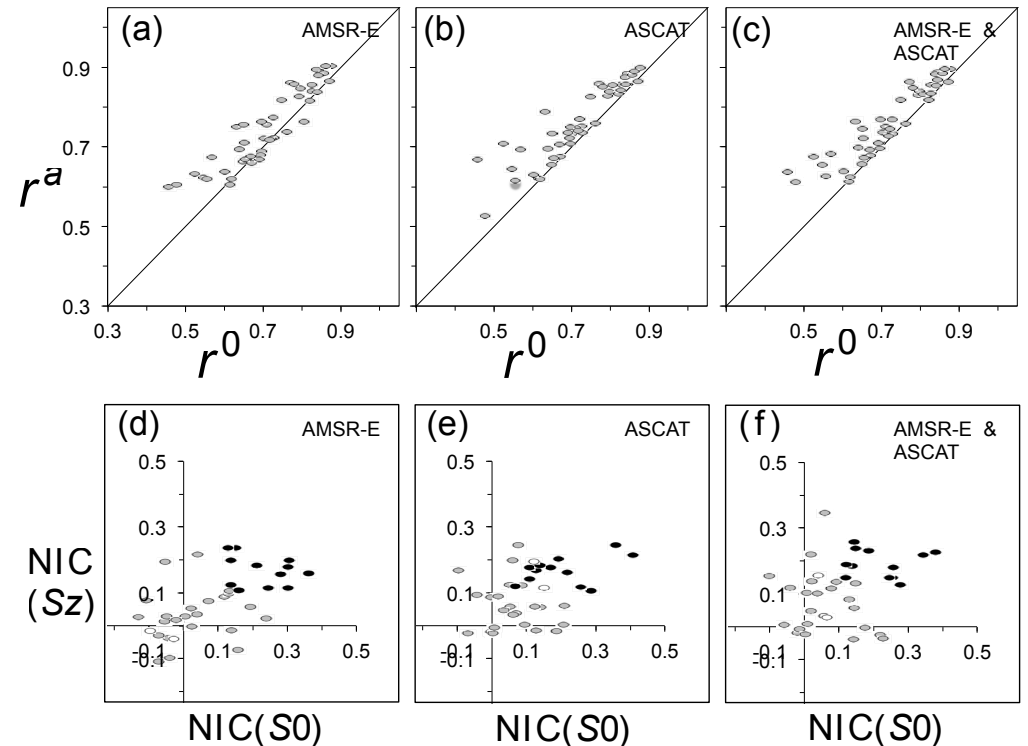

Figure 3. Correlation of AWRA-L simulations of top-layer SM against OzNet measurements before (open-loop $\left.r^{0}\right)$ and after assimilation $\left(r^{a}\right)$ of (a) AMSR-E, (b) ASCAT and (c) both satellite SM products jointly. Bottom panel shows the NIC statistic for top-layer $(S O)$ and shallow root-zone estimation $(\mathrm{Sz})$ after the assimilation of (a) AMSR-E; (b) ASCAT and (c) both SM products jointly.

found a cluster of sites around Kyeamba and Adelong where the assimilation of either AMSR-E or ASCAT seems to have little or no affect. These sites have higher TC errors than others, likely due to higher vegetation density in the region. Interestingly, ASCAT fares better for these sites.

AWRA-L estimates of shallow root-zone SM were created by summing the model's water storages in the top and shallow-root soil layers. Here we evaluate the ensemble mean shallow root-zone SM against the 37 OzNet sites where 0-30 cm measurements are available. We evaluate improvement of the assimilation results by computing the normalized information contribution (NIC) statistic (Kumar, et al, 2009) which measures improvement in correlation relative to open-loop simulations as $N I C=\left(r^{a}-r^{0}\right) /\left(1-r^{0}\right)$. We computed NIC statistics for the shallow root-zone SM (NIC $(S z)$ ) and, for comparison, the top-layer SM estimates (NIC $(S 0)$ ). These are displayed for each of the assimilation experiments in Figure 3 (bottom panel). Points in the top-right quadrant indicate that SM assimilation improve both top-layer and shallow root-zone estimation; points in the bottom left neither shallow root-zone nor top layer are improved through assimilation of SM. Overall we see that SM assimilation improve both the top-layer and shallow root-zone estimation.

Twelve of the 37 OzNet sites show improvements in both shallow root-zone and top-layer SM with NIC's greater than 0.1 common to all assimilation experiments. The sites, identified in Figures $3 \mathrm{~d}-\mathrm{f}$ as black dots, correspond to OzNet sites M1, 2, 5 and 6; Y1, 4-7, 11-12; and K10. AMSR-E has the lowest TC errors for the Yanco sites, which generally possess lower fractions of vegetation cover compared with other OzNet sites. Two of the six sites where the assimilation of AMSR-E alone did not improve top-layer nor shallowroot zone SM, displayed in Figures 3d-f as white dots, correspond to Kyeamba sites K12 and K14. We noticed for these two sites, TC errors were equal in magnitude ( 0.11 in relative wetness units). ASCAT assimilation, however, improved results for both top and shallow root-zone layers, perhaps once again indicating better performance of the ASCAT SM in areas of moderate vegetation density. The joint assimilation of AMSR-E and ASCAT results in improvements but substantially dampened compared to ASCAT alone because of the equal weighting (errors) associated with the respective data sets at these sites.

\section{DISCUSSION AND CONCLUSIONS}

Our evaluation of AWRA-L soil moisture (SM) estimation over the OzNet Murrumbidgee network of in situ sensors revealed the model to be performing very well, with correlations for both top and shallow root-zone generally between $0.60-0.89$. Model performance is enhanced (i.e. up to $20-30 \%$ increase in correlation) through the assimilation of remotely-sensed soil moisture products (AMSR-E and ASCAT), with the greatest improvements occurring for sites where the satellite products have low error. The AMSR-E product had lower errors in the less vegetated parts of the study area. Joint assimilation of AMSR-E and ASCAT 
improves SM at sites with a moderate level of vegetation cover, where the AMSR-E data alone had no impact or slightly degrade performance.

Adequate ensemble spread and accurate observation error characterisation are critical factors to the successful application of assimilation. In addition to the modelling output, we have examined the innovation statistics (differences between observations and model forecasts) that revealed possible suboptimal performance across some parts of Australia (results not shown here). This may be due to insufficient ensemble spread resulting from smaller than needed perturbation on the rainfall forcing. Our plan in the immediate future is to implement spatially varying relative error on the rainfall data and to incorporate a model error (a feature lacking in the current algorithm). Triple collocation errors for AMSR-E and ASCAT appear to be consistent with experience and our understanding of the pros and cons of satellite-derived SM. In future we will introduce stringent quality control measures on the satellite SM products.

The complementarity between AMSR-E and ASCAT has been observed before (e.g. Draper et al., 2012) and indeed is the basis of upcoming combined active-passive SM sensing systems (SMAP mission). Further investigation is needed to identify where (and when) satellite data should be assimilated. Our first steps towards this is to extend our evaluation of the model to other parts of Australia, particularly through exploring the use of emerging proximal sensing technologies, such as cosmic ray soil moisture detectors (COSMOS, http://cosmos.hwr.arizona.edu), as well as evaluating a wider variety of water balance terms.

\section{Acknowledgements}

This work was conducted under the Water Information Research and Development Alliance between CSIRO Water for a Healthy Country and the Bureau of Meteorology. We a grateful to our CSIRO colleagues Garth Warren, Tim Raupach and Dr Edward King for sourcing and preparing the satellite data for this investigation.

\section{REFERENCES}

Brocca, L., F. Melone, T. Moramarco, W. Wagner, V. Naeimi, Z. Bartalis, and S. Hasenauer. (2010) Improving runoff prediction through the assimilation of the ASCAT soil moisture product, Hydrology and Earth System Sciences, 14(10), 1881-1893, doi:10.5194/hess-14-1881-2010

Draper, C.S., R. H. Reichle, G. J. M. De Lannoy, and Q. Liu, (2012) Assimilation of passive and active microwave soil moisture retrievals, Geophysical Research Letters, 39, L04401

Caires, S., and A. Sterl, (2003). Validation of ocean wind and wave data using triple collocation, J. Geophys. Res., 108(C3), 3098,doi:10.1029/2002JC001491.

Evensen, G. (2003). The Ensemble Kalman Filter: theoretical formulation and practical implementation. Ocean Dynamics, 53(4), 343-367. doi:10.1007/s10236-003-0036-9

Jones, D. A., W. Wang, and R. Fawcett, (2009). High-quality spatial climate data-sets for Australia, Australian Meteorological and Oceanographic Journal, 58, 233-248.

Koster, R. D., Z. Guo, R. Yang, P.A. Dirmeyer, K. Mitchell, and M. J. Puma, (2009). On the Nature of Soil Moisture in Land Surface Models. Journal of Climate, 22(16), 4322-4335. doi:10.1175/2009JCLI2832.1

Kumar, S. V., RH. Reichle, R.D. Koster, W.T. Crow, and C.D. Peters-Lidard, (2009). Role of Subsurface Physics in the Assimilation of Surface Soil Moisture Observations. Journal of Hydrometeorology, 10(6), 1534-1547.

Liu, Y. Y., R.M. Parinussa, W.A. Dorigo, R.A.M. De Jeu, W. Wagner, A.I.J.M. Van Dijk, M.F. McCabe, et al. (2011). Developing an improved soil moisture dataset by blending passive and active microwave satellitebased retrievals. Hydrology and Earth System Sciences, 15(2), 425-436. doi:10.5194/hess-15-425-2011

Owe, M., R. De Jeu, and T. Holmes, (2008). Multisensor historical climatology of satellite-derived global land surface moisture. Journal of Geophysical Research, 113(F1), 1-17. doi:10.1029/2007JF000769

Reichle, R.H.. and R. D. Koster. (2005). Global assimilation of satellite surface soil moisture retrievals into the NASA Catchment land surface model', Geophysical Research Letters, 32, L02404

Smith, A. B., J.P. Walker, A.W. Western, R.I. Young, K.M. Ellett, R.C. Pipunic, R.B. Grayson, et al. (2012). The Murrumbidgee soil moisture monitoring network data set. Water Resources Research, 48(7), 1-6.

Van Dijk, A. I. J. M. (2010). AWRA Technical Report 3, Landscape Model (version 0.5) Technical Description, CSIRO Water for a Healthy Country Flagship, www.clw.csiro.au/publications/waterforahealthycountry/2010/wfhc-aus-water-resources-assessment-system.pdf

Wagner, W., G. Lemoine, and H. Rott, (1999). A method for estimating soil moisture from ERS Scatterometer and soil data, Rem. Sensing. Environ., 70, 191-207. 